\title{
Unternehmenserfolg durch Nachhaltigkeit - Reifegrad- und Vorgehensmodell zum Aufbau eines datenbasierten Nachhaltigkeitsmanagements
}

\author{
Eva Glanze $\mathbb{D} \cdot$ Markus Nüttgens • Will Ritzrau
}

Eingegangen: 9. September 2020 / Angenommen: 3. Dezember 2020 / Online publiziert: 15. Dezember 2020

(C) Der/die Autor(en) 2020

Zusammenfassung Im Verlauf der letzten vier Jahrzehnte ist Nachhaltigkeit aufgrund vielfältiger nationaler und internationaler Initiativen zu einem zentralen Begriff auch in der Wirtschaftsinformatik geworden. Sie wird auf Basis des DreiSäulen-Modells (Ökonomie, Ökologie, Soziales) operationalisiert und soll die Lebensgrundlage aktueller und zukünftiger Generationen sichern. Durch strengere gesetzliche Anforderungen, wachsenden Wettbewerbsdruck und die zunehmend von Nachhaltigkeitskriterien getriebenen Entscheidungen externer Interessengruppen sehen sich Unternehmen weltweit der zentralen Herausforderung gegenüber, Gewinnerzielung und Nachhaltigkeitskriterien in Einklang zu bringen. Eine wesentliche Grundlage hierfür bietet das datenbasierte betriebliche Nachhaltigkeitsmanagement. Das in diesem Beitrag vorgestellte Reifegradmodell synthetisiert und erweitert den aktuellen Stand der Forschung auf Basis einer Literaturrecherche. Es analysiert die organisationale Verwendung von Rahmenwerken, Kennzahlen und Steuerungswerkzeugen sowie die Reife des Nachhaltigkeitsdatenmanagements und die zu seiner Umsetzung verwendete IT-Architektur. Das Reifegrad- und das Vorgehensmodell sollen auch mittelständischen Unternehmen ein Instrumentarium bieten, um eine pragmatische Einschätzung des Status quo sowie des Entwicklungspotentials des eigenen Nachhaltigkeitsmanagements vorzunehmen.

\footnotetext{
E. Glanze $(\bowtie) \cdot$ M. Nüttgens

Hamburg Research Center for Information Systems (HARCIS), Universität Hamburg, Hamburg, Deutschland

E-Mail: eva.glanze@uni-hamburg.de

M. Nüttgens

E-Mail: markus.nuettgens@uni-hamburg.de

W. Ritzrau

SAP SE, Hochschule Fresenius, Heidelberg, Deutschland

E-Mail: will.ritzrau@hs-fresenius.de
} 
Schlüsselwörter Nachhaltigkeit · Nachhaltigkeitsmanagement · Reifegradmodell · Vorgehensmodell · KMU

\title{
Corporate Success through Sustainability - Maturity and Procedure Model for Setting Up a Data-Based Sustainability Management
}

\begin{abstract}
In the course of the last four decades, sustainability has also become a central concept in business information systems engineering, thanks to a variety of national and international initiatives. It is operationalised on the basis of the threepillar-model (social, ecological and economic issues) and aims to secure the basis of life for current and future generations. As a result of stricter legal requirements, growing competitive pressure and increasingly sustainability-driven decisions by external stakeholders, companies around the world are facing the central challenge of reconciling profit generation and sustainability criteria. Herefore, data-based corporate sustainability management provides a key foundation. The maturity model presented in this article synthesises and expands the current state of research based on a literature review. It analyses the organisational use of frameworks, key performance indicators and management tools as well as the maturity of a company's sustainability data management and the IT architecture used for its implementation. The maturity as well as the procedure model are intended to also provide mediumsized companies with a set of instruments for a pragmatic assessment of the status quo and the development potential of their own sustainability management.
\end{abstract}

Keywords Sustainability · Sustainability management $\cdot$ Maturity model · Procedure model $\cdot$ SME

\section{Einleitung und Grundlagen}

Nachhaltigkeit wird auch für die Wirtschaftsinformatik als zentrales Forschungsfeld im 21. Jahrhundert genannt (Nüttgens et al. 2011). Eine zentrale Herausforderung ist die Operationalisierung entlang der Wertschöpfungsketten und deren organisatorische und prozessuale Verankerung. Gründe sind ihre Multi-Dimensionalität, die Notwendigkeit der Langzeitbetrachtung ihrer Wirkeffekte und ihr Einfluss auf allen Ebenen naturgegebener und von Menschen geschaffener Systeme (Becker et al. 2015). Einen Einblick in ihre Entwicklungsgeschichte bietet Schneider (2015). Tab. 1 zeigt einen Überblick über nationale und internationale Initiativen der letzten 40 Jahre, welche die Relevanz der Nachhaltigkeit für deutsche Unternehmen maßgeblich beeinflusst haben. Es wurden nur Initiativen ausgewählt, welche dem ganzheitlichen Anspruch genügen (Ökonomie, Ökologie, Soziales).

Der 1987 von der Weltkommission für Umwelt und Entwicklung der Vereinten Nationen (United Nations; UN) veröffentlichte Brundtland-Bericht definiert Aktivitäten als nachhaltig, welche die Bedürfnisse der Gegenwart befriedigen ohne zugleich die Fähigkeit zukünftiger Generationen einzuschränken, ihre Bedürfnisse zu befriedigen (United Nations 1987). Er gilt als Ursprung des aktuellen Nachhaltigkeitsverständnisses. 1994 etablierte John Elkington die sogenannte Triple-Bottom- 
Tab. 1 Übersicht ausgewählter nationaler und internationaler Nachhaltigkeitsinitiativen der letzten 40 Jahre, welche auf die parallele Erreichung ökonomischer, ökologischer und sozialer Ziele ausgerichtet sind

\begin{tabular}{|c|c|c|c|}
\hline Jahr & Initiative & Abkürzung & Erläuterung \\
\hline 1987 & $\begin{array}{l}\text { Weltkommission für Umwelt und } \\
\text { Entwicklung der Vereinten Nationen }\end{array}$ & - & $\begin{array}{l}\text { Brundtland-Bericht gilt als Ursprung des } \\
\text { aktuellen Nachhaltigkeitsverständnisses }\end{array}$ \\
\hline 1998 & $\begin{array}{l}\text { Enquete-Kommission „Schutz des } \\
\text { Menschen und der Umwelt“ des } \\
\text { Deutschen Bundestages }\end{array}$ & - & $\begin{array}{l}\text { Abschlussbericht operationalisiert Nach- } \\
\text { haltigkeit mithilfe des Drei-Säulen-Mo- } \\
\text { dells }\end{array}$ \\
\hline 2000 & $\begin{array}{l}\text { Globaler Pakt der Vereinten Natio- } \\
\text { nen }\end{array}$ & UNGC & $\begin{array}{l}\text { Veröffentlichung von } 10 \text { freiwilligen } \\
\text { Nachhaltigkeitsprinzipien für Unterneh- } \\
\text { men }\end{array}$ \\
\hline 2006 & Global Reporting Initiative & GRI & $\begin{array}{l}\text { Veröffentlichung des ersten globalen } \\
\text { Rahmenwerkes zur Nachhaltigkeitsbe- } \\
\text { richterstattung }\end{array}$ \\
\hline 2010 & Deutscher Nachhaltigkeitskodex & DNK & $\begin{array}{l}\text { Nationaler Standard zur Nachhaltigkeits- } \\
\text { berichterstattung }\end{array}$ \\
\hline 2015 & $\begin{array}{l}\text { Ziele für nachhaltige Entwicklung } \\
\text { der Vereinten Nationen }\end{array}$ & SDGs & $\begin{array}{l}17 \text { Ziele mit } 169 \text { Unterzielen, die bis } \\
2030 \text { erreicht werden sollen }\end{array}$ \\
\hline 2017 & $\begin{array}{l}\text { Corporate-Social-Responsibility- } \\
\text { Richtlinie-Umsetzungsgesetz des } \\
\text { Deutschen Bundestages }\end{array}$ & CSR-RUG & $\begin{array}{l}\text { Gesetzliche Berichtspflicht für nicht-fi- } \\
\text { nanzielle Aspekte für Unternehmen mit } \\
\text { mehr als } 500 \text { Mitarbeitenden }\end{array}$ \\
\hline 2019 & Value-Balancing-Alliance & VBA & $\begin{array}{l}\text { Veröffentlichung eines globalen Nach- } \\
\text { haltigkeitsrechnungslegungsstandards bis } \\
2022 \text { geplant }\end{array}$ \\
\hline
\end{tabular}

Line (People, Planet, Profit, siehe Elkington [1994]). Der Abschlussbericht der Enquete-Kommission ,Schutz des Menschen und der Umwelt“ des Deutschen Bundestages führte dieses Drei-Säulen-Modell (Ökonomie, Ökologie, Soziales) als grundlegende Operationalisierung von Nachhaltigkeit 1998 in Deutschland ein.

2000 forderte die UN mit dem Globalen Pakt (United Nations Global Compact; UNGC) Unternehmen weltweit auf, sich freiwillig zu 10 Nachhaltigkeitsprinzipien zu verpflichten. Der allgemein gehaltene UNGC stand jedoch in der Kritik, keine belegbaren Veränderungen zu bewirken. Um die organisationale Nachhaltigkeitsleistung nachweislich messbarer und vergleichbarer zu machen, veröffentlichte die Global Reporting Initiative (GRI) im Jahr 2006 das erste globale Rahmenwerk von Finanz-, Sozial- und Umweltkennzahlen als Grundlage der Nachhaltigkeitsberichterstattung. Einige dieser GRI-Indikatoren bilden einen Teil der nicht-finanziellen Leistungsindikatoren des Deutschen Nachhaltigkeitskodexes (DNK), der seit 2010 als nationaler Standard zur Nachhaltigkeitsberichterstattung für Unternehmen gilt.

Als Nachfolger der 2010 veröffentlichten Millenniums-Entwicklungsziele wurde 2015 die bislang umfassendste globale Nachhaltigkeitsinitiative von den UN verabschiedet: Die 17 Ziele für nachhaltige Entwicklung (Sustainable Development Goals; SDGs), die bis 2030 erreicht werden sollen. Getrieben wird die durch 169 Unterziele und ca. 270 Indikatoren gemessene Zielerreichung durch die aktive Zusammenarbeit von öffentlichen Institutionen und Privatwirtschaft. Für Unternehmen werden die GRI-Standards empfohlen, um Nachhaltigkeitsinformationen in ihre Be- 
richterstattung zu integrieren. Diese sind inzwischen ebenso wie der UNGC auf die Erreichung der SDGs ausgerichtet.

Abgesehen von der Einhaltung gesetzlicher Regularien zum Umweltmanagement und Arbeitsschutz beruhte bis zum Ende der 2010-Jahre das Nachhaltigkeitsengagement deutscher Unternehmen auf Freiwilligkeit. Leitlinien bei der Umsetzung in diesen Bereichen bieten bis heute internationale Standards wie z. B. ISO 14001 (definiert Anforderungen für ein Umweltmanagementsystem) oder das von der Europäischen Union (EU) entwickelte „Eco-Management and Audit Scheme“ (EMAS), welches die Erfassung der Umweltleistung zertifiziert. Das Jahr 2017 markierte eine Zäsur: Durch die Umsetzung der EU-CSR-Richtlinie durch das CSR-RichtlinieUmsetzungsgesetz (CSR-RUG) führte der Deutsche Bundestag eine Berichtspflicht für nicht-finanzielle Aspekte für Unternehmen mit mehr als 500 Mitarbeitenden ein. Sie können den Nachweis ihrer Nachhaltigkeitsleistung durch wählbare Leistungsindikatoren erbringen; es wird die Nutzung des DNK empfohlen.

2019 wurde mit der Value-Balancing-Alliance (VBA) die erste global aktive, von Unternehmen und nicht von der Politik initiierte Nachhaltigkeitsinitiative gegründet. Diese Nichtregierungsorganisation wird von den vier größten Wirtschaftsprüfungen pro bono beraten und u. a. durch die EU, die GRI, den UNGC und von Universitäten unterstützt. Ziel ist die Entwicklung eines weltweiten Rechnungslegungsstandards, der über die Bewertung der positiven und negativen Auswirkungen der Geschäftstätigkeit von Unternehmen hinaus ganzheitlich die Berichterstattung integriert und die operative Unternehmenssteuerung konsistent und vergleichbar unterstützt.

Es wird deutlich, dass Nachhaltigkeit trotz der eingangs genannten Schwierigkeiten der konkreten Mess- und Bewertbarkeit von einem unscharf konturierten Begriff $\mathrm{zu}$ einem operationalisierbaren Konstrukt heranreift. Im Zuge dessen steigen auch die gesetzlichen Anforderungen und die gesellschaftliche Erwartungshaltung an Organisationen. Die Verankerung von Nachhaltigkeit in allen Kernprozessen bietet zugleich die Chance, den Unternehmenserfolg zu steigern (Dao et al. 2011). Nach Ritzrau (2017) liegen die Treiber hierfür u. a. in höherem Mitarbeiterengagement, gesteigerter Prozesseffizienz und größerer Innovationsfähigkeit. Unternehmenserfolg lässt sich als ganzheitliche Zielerreichung, beispielsweise mithilfe der Sustainability-Balanced-Scorecard, steuern und messen (Figge et al. 2002). Hierzu wird die Finanz-, Umwelt- (z.B. Treibhausgasemissionen, Energieverbrauch, Recyclingrate) und Sozialperspektive (z. B. Mitarbeiterengagement, Gesundheit und Sicherheit, Mitarbeiterbindung) integrativ betrachtet.

Die Motivation zur nachhaltigeren Geschäftstätigkeit wird folglich durch drei Entwicklungen getrieben:

- strengere gesetzliche Anforderungen in Bezug auf die Erbringung und den Nachweis der eigenen Nachhaltigkeitsleistung

- höherer Wettbewerbsdruck durch Vorreiterunternehmen, die eine ganzheitliche Unternehmensführung zur Optimierung ihrer Ressourcenproduktivität und Innovationskraft verfolgen

- zunehmend von Nachhaltigkeitskriterien getriebene Entscheidungen externer Interessengruppen wie Investoren- und Kundschaft sowie Arbeitnehmern. 


\section{Reifegradmodell für ein datenbasiertes betriebliches Nachhaltigkeitsmanagement}

Das nachfolgende Reifegradmodell basiert auf konzeptionellen Überlegungen, abgesichert durch eine Literaturrecherche. Hierzu wurden die etablierten internationalen Zeitschriften der Wirtschaftsinformatik (,Basket of Eight“) sowie die führende deutsche Zeitschrift BISE (Business \& Information Systems Engineering) durchsucht. Darüber hinaus wurden die Datenbanken Business Source Complete via EBSCO Host und SpringerLink herangezogen. Als relevant wurden Ergebnisse bewertet, die das betriebliche Nachhaltigkeitsmanagement aus gesamtunternehmerischer Sicht behandelten. Auf Grundlage weniger erster Treffer wurde die Suche im Schneeballverfahren fortgesetzt. Insgesamt konnten fünf Reifegradmodelle im Themengebiet identifiziert werden (El-Gayar und Fritz 2006; Müller-Christ und Giesenbauer 2019; Niemöller et al. 2015; Schneider 2015; Warnecke et al. 2018). Auffällig war, dass diese Arbeiten Aspekte der Umsetzung wie Rahmenwerke, Kennzahlen, Datenmanagement und IT-Architektur nicht thematisierten.

Tab. 2 zeigt auf der Grundlage von fünf Reifegradstufen das resultierende Reifegradmodell für ein datenbasiertes betriebliches Nachhaltigkeitsmanagement. Die Reifegrade wurden auf Basis von Ritzrau (2017) sowie Hogrebe und Nüttgens (2009) mit den Kategorien Beispielkennzahlen und Nachhaltigkeitsdatenmanagement kon-

Tab. 2 Reifegradmodell für ein datenbasiertes betriebliches Nachhaltigkeitsmanagement (eigene Darstellung auf Basis von Dao et al. 2011; El-Gayar und Fritz 2006; Hogrebe und Nüttgens 2009; Müller-Christ und Giesenbauer 2019; Niemöller et al. 2015; Ritzrau 2017; Schneider 2015; sowie Warnecke et al. 2018)

\begin{tabular}{|c|c|c|c|c|c|}
\hline & 1.0 & 2.0 & 3.0 & 4.0 & 5.0 \\
\hline $\begin{array}{l}\text { Selbstver- } \\
\text { ständnis }\end{array}$ & Skeptiker & Opportunist & Gestalter & Wertschöpfer & Innovator \\
\hline $\begin{array}{l}\text { Umsetzungs- } \\
\text { fokus }\end{array}$ & $\begin{array}{l}\text { Ausschließlich } \\
\text { Finanzen }\end{array}$ & $\begin{array}{l}\text { Separate Be- } \\
\text { trachtung von } \\
\text { Finanzen und } \\
\text { Nachhaltigkeit }\end{array}$ & $\begin{array}{l}\text { Integrative Be- } \\
\text { trachtung von } \\
\text { Finanzen und } \\
\text { Nachhaltigkeit }\end{array}$ & $\begin{array}{l}\text { Innerbetrieb- } \\
\text { liches nachhal- } \\
\text { tiges Manage- } \\
\text { ment }\end{array}$ & $\begin{array}{l}\text { Überbetriebliches } \\
\text { nachhaltiges } \\
\text { Management }\end{array}$ \\
\hline $\begin{array}{l}\text { Nachhaltig- } \\
\text { keitsrahmen- } \\
\text { werk }\end{array}$ & $\begin{array}{l}\text { Handels- } \\
\text { gesetzbuch }\end{array}$ & $\begin{array}{l}\text { Globaler Pakt } \\
\text { der Vereinten } \\
\text { Nationen }\end{array}$ & $\begin{array}{l}\text { Deutscher Nach- } \\
\text { haltigkeitskodex }\end{array}$ & $\begin{array}{l}\text { Standards } \\
\text { der Global } \\
\text { Reporting } \\
\text { Initiative }\end{array}$ & $\begin{array}{l}\text { Standard der } \\
\text { Value Balancing } \\
\text { Alliance }^{\mathrm{a}}\end{array}$ \\
\hline $\begin{array}{l}\text { Beispiel- } \\
\text { kennzahlen }\end{array}$ & $\begin{array}{l}\text { Deckungs- } \\
\text { beitrag }\end{array}$ & $\begin{array}{l}\text { Gesamtemissionen, } \\
\text { Unfallhäufigkeit }\end{array}$ & $\begin{array}{l}\text { Recyclingrate, } \\
\text { Zufriedenheit der } \\
\text { Mitarbeitenden }\end{array}$ & $\begin{array}{l}\mathrm{CO}_{2} \text {-Neutralität, } \\
\text { Mitarbeiterbin- } \\
\text { dung }\end{array}$ & $\begin{array}{l}\text { Valuierte Exter- } \\
\text { nalitäten }\end{array}$ \\
\hline $\begin{array}{l}\text { Steuerungs- } \\
\text { werkzeug }\end{array}$ & $\begin{array}{l}\text { Kosten- und } \\
\text { Leistungs- } \\
\text { rechnung }\end{array}$ & $\begin{array}{l}\text { Prozesseffizienz- } \\
\text { analyse }\end{array}$ & $\begin{array}{l}\text { Sustainability- } \\
\text { Balanced- } \\
\text { Scorecard }\end{array}$ & Szenarioanalyse & $\begin{array}{l}\text { Maschinelles } \\
\text { Lernen }\end{array}$ \\
\hline $\begin{array}{l}\text { Nachhaltig- } \\
\text { keitsdaten- } \\
\text { management }\end{array}$ & $\begin{array}{l}\text { Ad hoc } \\
\text { Datenerhe- } \\
\text { bung und } \\
\text {-auswertung }\end{array}$ & $\begin{array}{l}\text { Vielfalt der } \\
\text { Vorgehensweisen }\end{array}$ & $\begin{array}{l}\text { Einzelne zertifi- } \\
\text { zierte Bereiche, } \\
\text { hohe Datenqualität }\end{array}$ & $\begin{array}{l}\text { Automatisiertes, } \\
\text { auditiertes } \\
\text { System }\end{array}$ & $\begin{array}{l}\text { Fortlaufende } \\
\text { Optimierung, } \\
\text { hohe ethische } \\
\text { Standards }\end{array}$ \\
\hline IT-Architektur & $\begin{array}{l}\text { Standard- } \\
\text { Informations- } \\
\text { systeme (IS) }\end{array}$ & $\begin{array}{l}\text { Isolierte, abtei- } \\
\text { lungsspezifische } \\
\text { IS }\end{array}$ & $\begin{array}{l}\text { Standortweit } \\
\text { integrierte IS }\end{array}$ & $\begin{array}{l}\text { Organisationsweit } \\
\text { integriertes, } \\
\text { „grünes“ IS }\end{array}$ & $\begin{array}{l}\text { Wertschöpfungs- } \\
\text { zyklusweit inte- } \\
\text { griertes IS }\end{array}$ \\
\hline
\end{tabular}

${ }^{\mathrm{a} V e r o ̈ f f e n t l i c h u n g ~ f u ̈ r ~ d a s ~ J a h r ~} 2022$ angekündigt 
kretisiert, die Kategorie IT-Architektur basiert auf Ausführungen von Dao et al. (2011).

\subsection{Reifegrad 1.0}

Im Reifegrad 1.0 entspricht das unternehmerische Selbstverständnis dem eines Nachhaltigkeitsskeptikers. Nachhaltigkeit wird als weitgehend irrelevant betrachtet, der Fokus im Unternehmen liegt ausschließlich auf finanziellem Erfolg.

Insgesamt ist das Unternehmen in seiner Geschäftstätigkeit kurzfristig orientiert sein langfristiges Innovationspotential bleibt so unzureichend genutzt. Ohne ein systematisches Risikomanagement kann es durch unvorhergesehene Ereignisse immer wieder in existenzbedrohende Krisen kommen.

Je nach Unternehmensgröße beschäftigen sich nur die notwendigerweise involvierten Mitarbeitenden rudimentär mit dem Thema Nachhaltigkeit, sofern überhaupt gesetzliche Berichtspflichten bestehen (z.B. CSR-RUG). Meist wird ausschließlich ein Finanzbericht veröffentlicht, der die grundlegende unternehmerische Verantwortungsübernahme im Sinne der gesetzlichen Bestimmungen des Handelsgesetzbuches beschreibt.

Die im gesamten Unternehmen dominierende Kennzahl für die Unternehmenssteuerung und die Leistungsbewertung ist der erwirtschaftete Deckungsbeitrag. Wenn überhaupt Nachhaltigkeitsdaten erhoben und ausgewertet werden, erfolgt dies mithilfe von Standard-Informationssystemen ad hoc. Dieser Prozess ist unsicher, ressourcenintensiv und hängt in seiner Qualität stark von den ausführenden Personen ab.

\subsection{Reifegrad 2.0}

In Unternehmen des Reifegrades 2.0 entstehen Nachhaltigkeitsvorteile opportunistisch. Der Fokus der Ressourcenproduktivität geht mit einem geringeren Verbrauch natürlicher Ressourcen einher und wird in den meisten Fällen nicht mit Nachhaltigkeit in Verbindung gebracht.

Organisationsintern ist ein einheitliches Verständnis zum Thema Nachhaltigkeit nicht verankert. Eine systematische Erarbeitung, Bewertung, Umsetzung und Nachhaltung von Nachhaltigkeitsmaßnahmen im Sinne eines ganzheitlichen BusinessCases blieben bislang aus. Das Unternehmen setzt sich folglich dem Risiko aus, eines „Greenwashings“ bezichtigt zu werden. Durch die Dynamik sozialer Medien kann sich ein solcher Vorwurf bis zum Boykott hochschaukeln. Da das Unternehmen das Thema Nachhaltigkeit beim Risikomanagement nicht berücksichtigt, ist es sich dieses Reputationsrisikos nicht bewusst.

Nachhaltigkeitsthemen werden sporadisch nur bei subjektiv wahrgenommenem Mehrwert für das Unternehmen im Sinne eines philanthropischen Engagements verfolgt (z. B. Spenden für lokale Sozial- oder Umweltinitiativen). Die Unterzeichnung des UNGC wird gemeinsam mit den punktuellen CSR-Aktivitäten marketingwirksam in der Außendarstellung genutzt. Das Unternehmen veröffentlicht hierzu einen isolierten Nachhaltigkeitsbericht zusätzlich zur gesetzlich geforderten Berichterstattung. 
Die Organisation nutzt ökonomische und einzelne quantitative ökologische und soziale Kennzahlen wie z. B. Emissionen als Proxy für mögliche Steuern und Unfallhäufigkeit als arbeitsrechtliche Anforderung zur Unternehmenssteuerung. Das zur Entscheidungsfindung eingesetzte Werkzeug ist die vergangenheitsorientierte Prozesseffizienzanalyse. Die Prozesse des Umwelt- und Sozialdaten-Managements sind siloartig organisiert und an unverbindlichen Standards wie ISO 26000 orientiert. Die vorhandene Vielfalt der Vorgehensweisen in den unterschiedlichen Fachabteilungen erschwert die gesamtunternehmerische Nachhaltigkeitsdatenerhebung und -auswertung für Unternehmenssteuerung und -berichterstattung. Diese Aktivitäten sind folglich nicht zielorientiert verzahnt. Verstärkt wird das Problem durch die isolierten, abteilungsspezifischen Informationssysteme, die einen zeitintensiven, fehleranfälligen, manuellen Datenaustausch bedingen.

\subsection{Reifegrad 3.0}

Der Reifegrad 3.0 ist durch das Selbstverständnis als Gestalter charakterisiert. Die Führungsriege eines solchen Unternehmens übernimmt in Bezug auf Nachhaltigkeitsthemen in authentischer Weise eine Vorreiterrolle. Sie trägt innerbetrieblich die Verantwortung für die positiven und negativen Auswirkungen der Geschäftstätigkeit. Das Unternehmen ist hierzu mit externen Interessengruppen wie Investoren, Nichtregierungsorganisationen, Kundschaft und Lieferanten im kontinuierlichen Austausch und erweitert sein Netzwerk entsprechend seiner Ziele fortlaufend. Die durch eine langfristig orientierte, ganzheitliche Unternehmensführung getragene hohe Ressourcenproduktivität führt gemeinsam mit dem Engagement der Mitarbeitenden zu einer höheren Innovationsfähigkeit des Unternehmens.

Alle Organisationsmitglieder betrachten Finanzen und Nachhaltigkeit integrativ. Sie werden durch eine dedizierte Nachhaltigkeitsabteilung kontinuierlich in die Erreichung der durch die Geschäftsführung definierten, kommunizierten und nachgehaltenen ökonomischen, ökologischen und sozialen Unternehmensziele einbezogen. Der Wissensaustausch zum Thema Nachhaltigkeit wird über die Grenzen des Unternehmens hinaus vorangetrieben. Nachhaltigkeit wird so auf operativer, taktischer und strategischer Planungsebene zielgerichtet verfolgt und risikobezogen systematisch gemanagt. Sie ist damit Treiber für den Unternehmenserfolg.

Das Unternehmen veröffentlicht einen auditierten, integrierten Finanz- und Nachhaltigkeitsbericht auf Basis des DNK. Es engagiert sich in dieser Initiative als Vorreiter und trägt durch seine Erfahrungen zu ihrer Konkretisierung und Verbreitung bei.

Zur Steuerung des Unternehmens und als Messkriterien in internen Anreizsystemen werden beispielsweise die Recyclingrate und die Zufriedenheit der Mitarbeitenden genutzt, d.h. zusätzlich zu ökonomischen werden quantitative und qualitative ökologische und soziale Kennzahlen berücksichtigt. Als strategisches Steuerungswerkzeug wird die Sustainability-Balanced-Scorecard eingesetzt, um die interne Unternehmensanalyse mit einer externen Umfeldanalyse zu kombinieren und so Nachhaltigkeitsaspekte zu berücksichtigen (Figge et al. 2002). Das Unternehmen ist in seinen Entscheidungen datengetrieben; Unternehmenssteuerung und Berichterstattung erfolgen integriert und zukunftsorientiert. Dies wird durch die hohe Stan- 
dardisierung des Datenmanagements auf Basis dokumentierter Geschäftsprozesse, einem passgenauen betrieblichen Datenmodell, standortweit integrierten Informationssystemen und einer zentralisierten IT-Governance ermöglicht. Die Qualität der verwendeten Daten ist hoch, der Datenaustausch im Unternehmen verlässlich und sicher. Einzelne Bereiche des Nachhaltigkeitsdatenmanagements sind zertifiziert, z. B. besteht ein Umweltmanagementsystem nach ISO 14001.

\subsection{Reifegrad 4.0}

Das Selbstverständnis des Wertschöpfers kennzeichnet ein Unternehmen des Reifegrades 4.0. Seine Führungspersonen verfolgen ein ganzheitliches innerbetriebliches nachhaltiges Management, das auf der Verankerung von Nachhaltigkeit in allen Kernprozessen basiert. Alle Mitarbeitenden werden innerhalb der bestehenden Netzwerkorganisation systematisch und monetär in die Zielerreichung einbezogen. Es werden hierzu auf Basis von ganzheitlichen Business-Cases fortlaufend Ressourcen bereitgestellt.

Das Unternehmen lebt selbst glaubwürdig Nachhaltigkeit und stimuliert sie parallel dazu durch das eigene Portfolio von nachhaltigen Produkten und Dienstleistungen bei externen Interessengruppen. Auf diese Weise wird der langfristige Geschäftserfolg durch die Übernahme einer Multiplikatorrolle gesichert und das Geschäftspartner-Ökosystem kontinuierlich nachhaltiger. Das Unternehmen wird so seiner Verantwortung gerecht, zu einer ressourcenbewussten Wertschöpfung beizutragen; es erzielt seinen Unternehmenserfolg durch Nachhaltigkeit und ganzheitliche Steuerung.

Die hohe Automatisierung aller Geschäftsprozesse ermöglicht die ganzheitliche Unternehmenssteuerung sowie eine automatisierte, zielgruppenadaptierte Berichterstattung. Sie berücksichtigt finanzielle und nachhaltige Aspekte integrativ und ist entsprechend der GRI-Standards gestaltet.

In diesem Reifegrad bringt das Unternehmen soziale und ökologische Kennzahlen über Wirkzusammenhänge in Verbindung mit ökonomischen Kennzahlen. Es kann so die Auswirkungen einzelner Aktivitäten auf das operative Finanzergebnis quantifizieren. Das auf Basis einer Wesentlichkeitsanalyse identifizierte Kennzahlenset beinhaltet z. B. absolute, mittel- und langfristig orientierte Kennzahlen wie $\mathrm{CO}_{2}$ Neutralität oder eine bestimme Mitarbeiterbindung (Ritzrau 2017). Dieses Kennzahlen-Set ist für alle Organisationsmitglieder leitend. Das Unternehmen nutzt zur datengetriebenen, ganzheitlichen Entscheidungsfindung die automatisierte Szenarioanalyse. Sein Rückgrat bildet ein organisationsweit integriertes, ,grünes“ Informationssystem, das Mining- und Data-Science-Technologien nutzt. „Grün“ bedeutet hierbei, dass das System selbst nachweislich hohen Nachhaltigkeitsanforderungen entspricht (Molla et al. 2011).

\subsection{Reifegrad 5.0}

Das Unternehmen agiert im Reifegrad 5.0 als Innovator im Ökosystem. Die Grenzen seiner Geschäftsprozesse verschwimmen zunehmend, da es fortlaufend an der gemeinsamen Entwicklung innovativer Prozesse, Produkte, Dienstleistungen und 
Geschäftsmodelle beteiligt ist. Es unterstützt außerdem gesellschaftspolitische Nachhaltigkeitsinitiativen wie die SDGs.

Ein Unternehmen dieses Reifegrades verfolgt ein überbetriebliches nachhaltiges Management, das durch den systematischen, zielorientierten Austausch mit externen Interessengruppen getragen wird. Alle Organisationsmitglieder wirken proaktiv an den gemeinsam mit diesen externen Partnern definierten Zielen mit - Nachhaltigkeit ist systemimmanent.

Das Rahmenwerk des unternehmerischen Handelns und der Berichterstattung bildet der Standard der VBA, d.h. es erfolgt eine ganzheitliche, methodisch standardisierte Analyse und Bewertung aller positiven und negativen Auswirkungen der eigenen Geschäftstätigkeit. Als Kriterien sowohl für die Vergütung aller Mitarbeitenden als auch zur Messung des Unternehmenserfolges dienen valuierte Externalitäten.

Organisationsweit wird maschinelles Lernen eingesetzt, um Entscheidungen zusammen mit externen Interessengruppen im Sinne der gemeinsamen Ziele zu treffen. Das Datenmanagement wird hierzu fortlaufend optimiert und entspricht hohen ethischen Standards. Da interne mit externen Daten verknüpft sind, bilden eine von allen Partnern akzeptierte IT-Governance sowie die hohe Reife der gemeinsamen Geschäftsprozesse grundlegende Voraussetzungen für diesen Reifegrad. Alle Daten, die für die Abwicklung relevant sind, werden von einem wertschöpfungszyklusweit integrierten Informationssystem sinnhaft vollautomatisiert erfasst und ausgewertet. Fragen bezüglich Qualität, Erhebungsumfang und Verfügungsgewalt der Daten sowie mit Hinblick auf Anwendungsmethoden und Kostenverteilung dieses Systems sind vor seiner Implementierung partnerschaftlich geklärt worden.

\section{Vorgehensmodell zum Aufbau eines datenbasierten betrieblichen Nachhaltigkeitsmanagements}

Das vorgestellte Reifegradmodell ermöglicht es, den Status quo eines datenbasierten betrieblichen Nachhaltigkeitsmanagements einzuschätzen. Diese Standortbestimmung bildet den Ausgangspunkt für alle Aktivitäten zu seiner Entwicklung. Einzelne Stufen des Reifegradmodells können nicht übersprungen werden.

Die Basis für die erfolgreiche Umsetzung eines datenbasierten betrieblichen Nachhaltigkeitsmanagements bilden die eindeutige Positionierung der Führungspersonen und die kontinuierliche, systematische Involvierung der Mitarbeitenden. Nur so kann das Selbstverständnis des Unternehmens bis zum Innovator entwickelt werden. Der entsprechende Umsetzungsfokus des jeweiligen Reifegrades gibt hierfür das Leitmotiv vor. Für die Transformation sollte genug Zeit eingeplant werden. Meilensteine sollten in einem Zeitplan sichtbar festgehalten und Erfolge kommuniziert werden, um die Weiterentwicklung gemeinsam mit allen Mitarbeitenden voranzutreiben.

Das Unternehmen kann auf Basis dieses gemeinsamen Selbstverständnisses seine Anreizsysteme und Berichterstattung entsprechend des jeweiligen Rahmenwerkes und der zugehörigen Kennzahlen des gewünschten Reifegrades ausrichten und ein passendes Steuerungswerkzeug identifizieren. Um es anzuwenden, ist es erforderlich, dass das Unternehmen sein Datenmanagement entsprechend der neuen Anfor- 
derungen erweitert. Die hierzu benötigte IT-Architektur ergibt sich entsprechend. Ein passgenaues Projekt-, Wissens- und Risikomanagement sollte flankierend etabliert werden.

Durch das Reifegradmodell wird ebenfalls deutlich, welche Meilensteine das Unternehmen jeweils erreichen sollte; z. B. im Reifegrad 3.0 das Nachhaltigkeitsdatenmanagement und die Berichterstattung systematisieren und extern auditieren lassen. Die reifegradspezifischen Ergebnisse müssen jeweils evaluiert und kommuniziert werden, um zu bewerten, inwieweit die selbst gesteckten Ziele wie beispielsweise Produktivitätsgewinne, Reputationsverbesserungen oder höhere Innovationskraft erreicht worden sind. Der Zyklus kann dann erneut durchlaufen werden, bis der avisierte Reifegrad erreicht ist.

Zusätzliche Hilfestellung, wie der jeweils nächsthöhere Reifegrad konkret erreicht werden kann, gibt das in Abb. 1 dargestellte Vorgehensmodell. Es fasst auf Basis der Ausführungen dieses Artikels für jede Reifegradstufe vier konkrete Maßnahmen zusammen.

Setzt das Unternehmen die genannten Maßnahmen im Rahmen des beschriebenen ganzheitlichen Reifeprozesses um, richtet es seine Aktivitäten zunehmend extern aus. Es verändert seine Organisationsstrukturen, sein Produktportfolio und seine Partnerschaften entsprechend. Entscheidend für das Gelingen der Transformation ist es, Nachhaltigkeit durch ein stetig in Umfang und Detailgrad wachsendes

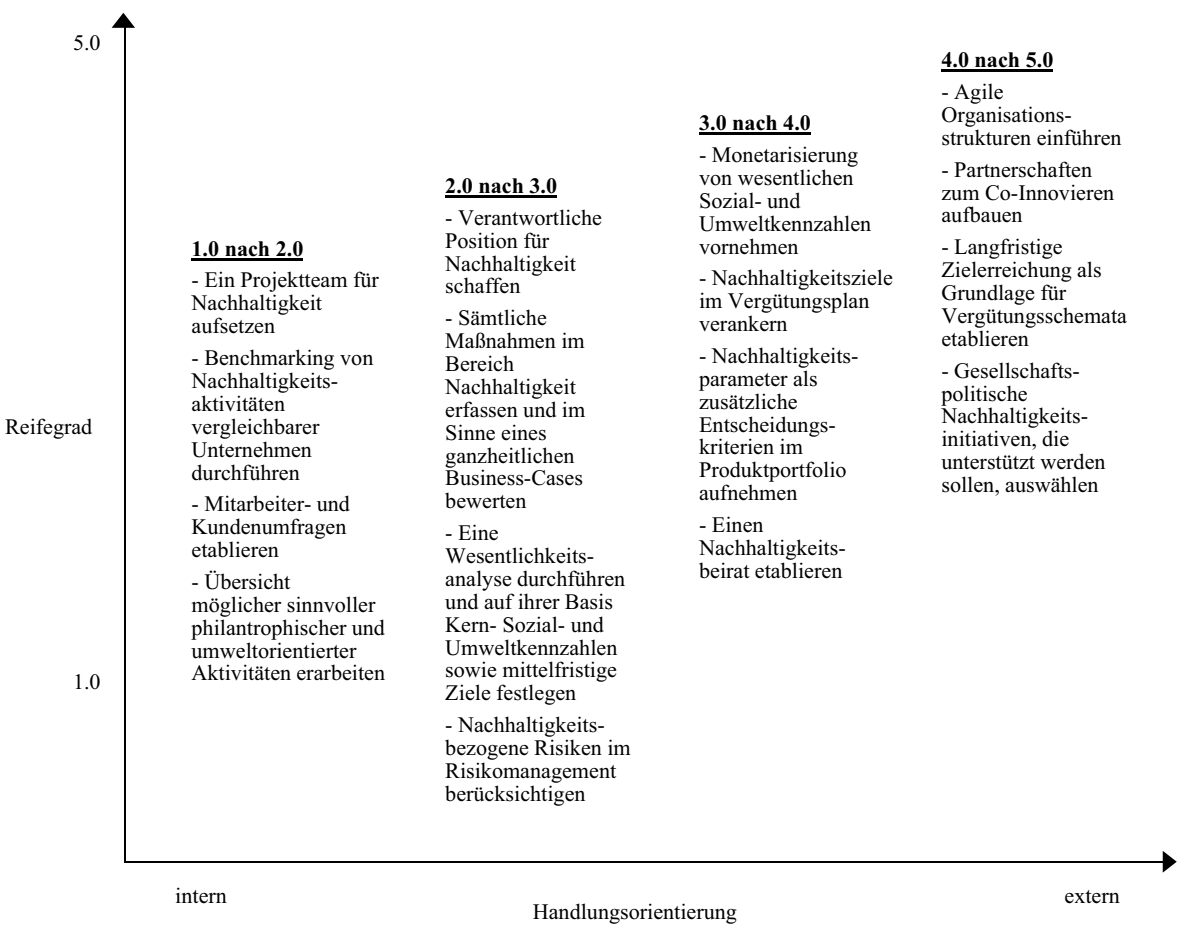

Abb. 1 Vorgehensmodell zum Aufbau eines datenbasierten betrieblichen Nachhaltigkeitsmanagements 
Kennzahlenset zu operationalisieren. Für seine Erhebung und Auswertung sollten die passenden Informationssysteme eingesetzt werden.

\section{Fazit}

Unternehmen sollten zur Sicherung ihrer Wettbewerbsfähigkeit ein datenbasiertes Nachhaltigkeitsmanagement mindestens des Reifegrades 3.0 aufbauen, um als Gestalter Nachhaltigkeit messbar und effizient umzusetzen. Durch zunehmende Prozessautomatisierung bei der Erfassung und Auswertung von Umwelt- und Sozialdaten können sie schließlich das Ziel erreichen, Nachhaltigkeit in ihren Kernprozessen zu verankern um als Wertschöpfer zu agieren. Sie sind dann mit Erreichung des Reifegrades 4.0 in der Lage, die eingangs genannten Herausforderungen zu meistern und damit:

- strengere gesetzliche Anforderungen in Bezug auf die Erbringung und den Nachweis ihrer Nachhaltigkeitsleistung effizient zu erfüllen, indem sie die Erhebung und Auswertung von Nachhaltigkeitsdaten organisationsweit automatisieren und auditieren lassen

- selbst Vorreiterunternehmen zu sein, die durch ganzheitliche Unternehmensführung ihre Ressourcenproduktivität und Innovationskraft fortlaufend optimieren, indem sie ihre Mitarbeitenden systematisch und monetär in die Zielerreichung einbeziehen

- von den zunehmend von Nachhaltigkeitskriterien getriebenen Entscheidungen externer Interessengruppen wie Investoren- und Kundschaft sowie Arbeitnehmern zu profitieren, indem sie ihr Angebotsportfolio nachhaltig ausrichten, ihre eigene Nachhaltigkeitsleistung fortlaufend steigern und deren Messwerkzeuge zielgruppengerecht offenlegen.

Überbetriebliches nachhaltiges Management als Innovator im Reifegrad 5.0 umzusetzen, ist eine Vision, an der Unternehmen ihre Aktivitäten heutzutage ausrichten sollten. In Anbetracht der nach wie vor bestehenden weltweiten ökonomischen und sozialen Ungleichheiten sowie der zunehmenden Auswirkungen des Klimawandels besteht akuter Handlungsbedarf, um ein zukunftsfähiges Wirtschaftssystem in einer stabilen Gesellschaft zu gestalten. Der vorliegende Beitrag mag als pragmatische Hilfestellung dienen, um eine nachhaltige Wirtschaftsweise erfolgreich umzusetzen.

Funding Open Access funding enabled and organized by Projekt DEAL.

Open Access Dieser Artikel wird unter der Creative Commons Namensnennung 4.0 International Lizenz veröffentlicht, welche die Nutzung, Vervielfältigung, Bearbeitung, Verbreitung und Wiedergabe in jeglichem Medium und Format erlaubt, sofern Sie den/die ursprünglichen Autor(en) und die Quelle ordnungsgemäß nennen, einen Link zur Creative Commons Lizenz beifügen und angeben, ob Änderungen vorgenommen wurden.

Die in diesem Artikel enthaltenen Bilder und sonstiges Drittmaterial unterliegen ebenfalls der genannten Creative Commons Lizenz, sofern sich aus der Abbildungslegende nichts anderes ergibt. Sofern das betreffende Material nicht unter der genannten Creative Commons Lizenz steht und die betreffende Handlung nicht nach gesetzlichen Vorschriften erlaubt ist, ist für die oben aufgeführten Weiterverwendungen des Materials die Einwilligung des jeweiligen Rechteinhabers einzuholen. 
Weitere Details zur Lizenz entnehmen Sie bitte der Lizenzinformation auf http://creativecommons.org/ licenses/by/4.0/deed.de.

\section{Literatur}

Becker C, Chitchyan R, Duboc L, Easterbrook S, Penzenstadler B, Seyff N, Venters C (2015) Sustainability design and software: the Karlskrona manifesto. 2015 IEEE/ACM 37th IEEE International Conference on Software Engineering. Bd. 2, S 467-476 https://doi.org/10.1109/ICSE.2015.179

Dao V, Langella I, Carbo J (2011) From green to sustainability: information technology and an integrated sustainability framework. J Strateg Inf Syst 20(1):63-79. https://doi.org/10.1016/j.jsis.2011.01.002

El-Gayar O, Fritz BD (2006) Environmental management information systems (EMIS) for sustainable development: a conceptual overview. CAIS 17(1):34. https://doi.org/10.17705/1CAIS.01734

Elkington J (1994) Towards the sustainable corporation: win-win-win business strategies for sustainable development. Calif Manage Rev 36(2):90-100. https://doi.org/10.2307/41165746

Figge F, Hahn T, Schaltegger S, Wagner M (2002) The sustainability balanced scorecard-linking sustainability management to business strategy. Bus Strategy Environ 11(5):269-284. https://doi.org/10. 1002/bse.339

Hogrebe F, Nüttgens M (2009) Business Process Maturity Model (BPMM): Konzeption, Anwendung und Nutzenpotenziale. HMD 46(2):17-25. https://doi.org/10.1007/BF03340339

Molla A, Cooper V, Pittayachawan S (2011) The green IT readiness (G-readiness) of organizations: an exploratory analysis of a construct and instrument. CAIS 29(1):67-96. https://doi.org/10.17705/1CAIS. 02904

Müller-Christ G, Giesenbauer B (2019) Konturen eines integralen Nachhaltigkeitsmanagements. In: Englert M, Ternès A (Hrsg) Nachhaltiges Management. Springer Gabler, Berlin, Heidelberg, S 231-248

Niemöller C, Bärtling N, Thomas O (2015) Nachhaltigkeit durch Hybride Wertschöpfung - Entwicklung eines Reifegradmodells. In: Cunningham DW, Hofstedt P, Meer K, Schmitt I (Hrsg) INFORMATIK 2015. Gesellschaft für Informatik, Bonn, S 427-442

Nüttgens M, Gadatsch A, Kautz K, Schirmer I, Blinn N (Hrsg) (2011) Governance and sustainability in information systems. Managing the transfer and diffusion of IT. IFIP WG 8.6 International Working Conference, Hamburg, Germany, September 22-24, 2011. Bd. 366. Springer, Berlin, Heidelberg

Ritzrau W (2017) Eine nachhaltige Strategie bei SAP. In: Wunder T (Hrsg) CSR und Strategisches Management. Springer Gabler, Berlin, Heidelberg, S 219-237

Schneider A (2015) Reifegradmodell CSR - Eine Begriffsklärung und -abgrenzung. In: Schneider A, Schmidpeter R (Hrsg) Corporate Social Responsibility. Springer Gabler, Berlin, Heidelberg, S 21-42

United Nations (1987) Report of the World Commission on Environment and Development: Our common future. http://www.un-documents.net/wced-ocf.htm. Zugegriffen: 08.06.2020

Warnecke D, Heyn J, Teuteberg F (2018) Nachhaltigkeit durch betriebliche Umweltinformationssysteme (BUIS)? Entwicklung und Evaluation eines Reifegradmodells für kleine und mittlere Unternehmen (KMU). In: Czarnecki C, Brockmann C, Sultanow E, Koschmider A, Selzer A (Hrsg) Workshops der INFORMATIK 2018 - Architekturen, Prozesse, Sicherheit und Nachhaltigkeit. Köllen, Bonn, S $145-156$ 